\title{
Face recognition using assemble of low frequency of DCT features
}

\author{
Raja Abdullah Raja Ahmad', Muhammad Imran Ahmad², Mohd Nazrin Md Isa ${ }^{3}$, Said \\ Amirul Anwar ${ }^{4}$ \\ ${ }^{1,2,4}$ School of Computer and Communication Engineering, Universiti Malaysia Perlis, Malaysia \\ ${ }^{3}$ School of Microelectronic Engineering, Universiti Malaysia Perlis, Malaysia
}

\section{Article Info}

Article history:

Received Dec 1, 2018

Revised Jan 20, 2019

Accepted Feb 21, 2019

\section{Keywords:}

DCT

Face recognition

Local feature extraction

Low frequency features

PCA

\begin{abstract}
Face recognition is a challenge due to facial expression, direction, light, and scale variations. The system requires a suitable algorithm to perform recognition task in order to reduce the system complexity. This paper focuses on a development of a new local feature extraction in frequency domain to reduce dimension of feature space. In the propose method, assemble of DCT coefficients are used to extract important features and reduces the features vector. PCA is performed to further reduce feature dimension by using linear projection of original image. The proposed of assemble low frequency coefficients and features reduction method is able to increase discriminant power in low dimensional feature space. The classification is performed by using the Euclidean distance score between the projection of test and train images. The algorithm is implemented on DSP processor which has the same performance as PC based. The experiment is conducted using ORL standard face databases the best performance achieved by this method is $100 \%$. The execution time to recognize 40 peoples is 0.3313 second when tested using DSP processor. The proposed method has a high degree of recognition accuracy and fast computational time when implemented in embedded platform such as DSP processor.
\end{abstract}

Copyright (C) 2019 Institute of Advanced Engineering and Science. All rights reserved.

\section{Corresponding Author:}

Raja Abdullah Raja Ahmad,

School of Computer and Communication Engineering,

Universiti Malaysia Perlis, School of Manufacturing Engineering Complex,

First Floor, Pauh Putra Campus, 02600 Arau, Perlis, Malaysia

Email: rajaabdullah@studentmail.unimap.edu.my

\section{INTRODUCTION}

Recognition system nowadays plays an important role for future interactions between humans and machines. Machines are able to finish jobs faster, in a more accurate and secure manner. The reliable methods of biometric personal identification already exist, for example, an iris or a fingerprint scanner. However, the identification of a person's facial is often effective without the participant's cooperation.

The challenge is now to develop a face recognition system with high accuracy, less complex, and minimal computational resources. Most of the face recognition algorithm utilizes holistic features to represent face image. Holistic features are captured from the whole face image. This method has several limitations especially when the images have illumination and pose variations. Local features are believed to be an effective way to extract the important features in the face image. Local features based on a Discrete Cosine Transform (DCT) are compute in several image regions. The image is separated into several regions that has different discrimination power. By selecting only small amount of features that produce the best performance, we are able to reduce processing time and minimize memory usage. The local features extraction approach is a process to preserve important information from certain face region, which makes this approach robust to differences in illumination and position [1]. 
The DCT has the ability to divide the information into a small number of coefficients on upper left side know as low frequency component. Compared to Karhunen Loeve Transform (KLT), DCT have advantage in potential smaller dimension, better processing time, and compatibility with encoded data such as jpeg. In this paper we utilize local information by using block base DCT to eliminate the effect of expression, illumination, pose and occlusion variations. Among all DCT coefficients, the first coefficient is called DC value that holds the entire energy as well as perceptual information of an image block.

Feature dimensional reduction based on Principle Component Analysis (PCA) is an efficient way to produce low dimensional feature space. This process further reduces feature dimensionality in the feature space after extracted using Discrete Cosine Transform (DCT) as stated in [2]. Principal Components are linear combinations of optimally weighted observed variables and is less complicated compared to Linear Discriminant Analysis (LDA) and Independent Component Analysis (ICA). Normally for this kind of feature extraction the classification process is done by using the Euclidean distance classifier. This approach will reduce the complexity of the classifier algorithm and produce better processing speed.

The implementation of the algorithm in DSP processor such as TMS320C6713 produces low-cost and fast processing time. Texas Instrument (TI) provided all required architecture that is suitable for many DSP applications such as image and signal analysis. The advantage of the C6x microprocessor is a very-longinstruction-word (VLIW), which can execute multiple processes in a single instruction. The C6x architecture is very well suited for numerically intensive calculations, where it is under the TMS320C6000 family. In the experiment analysis, TMS320C6713 is used because of its high performance and suitability for this kind of algorithm.

This paper proposes a new method of feature extraction to produce high discrimination feature space for real time face recognition system using local features extracted in face local regions. The main contributions of this paper are as follows:

a. Local feature extraction using low frequency information extracted in local region of face image to produce high discrimination feature.

b. Assemble of local feature using discrete cosine transform and principle components analysis to reduce noise and redundant information.

The propose method has been tested using ORL database. The success of using small features space is demonstrated in term of comparative performance against the other biometrics traits. The paper is organized as follows: Section 2 discusses about our proposed method; Section 3 contains the experimental analysis and discussion; and Section 4 is conclutions.

\section{FRAME WORK OF THE PROPOSED METHOD}

The propose multiple stage reduction process at feature level is able to produce small dimension of features. A matrix of low frequency features and simple statistical tool is used to capture the fundamental of statistical evidence in feature vector as shows in Figure 1.

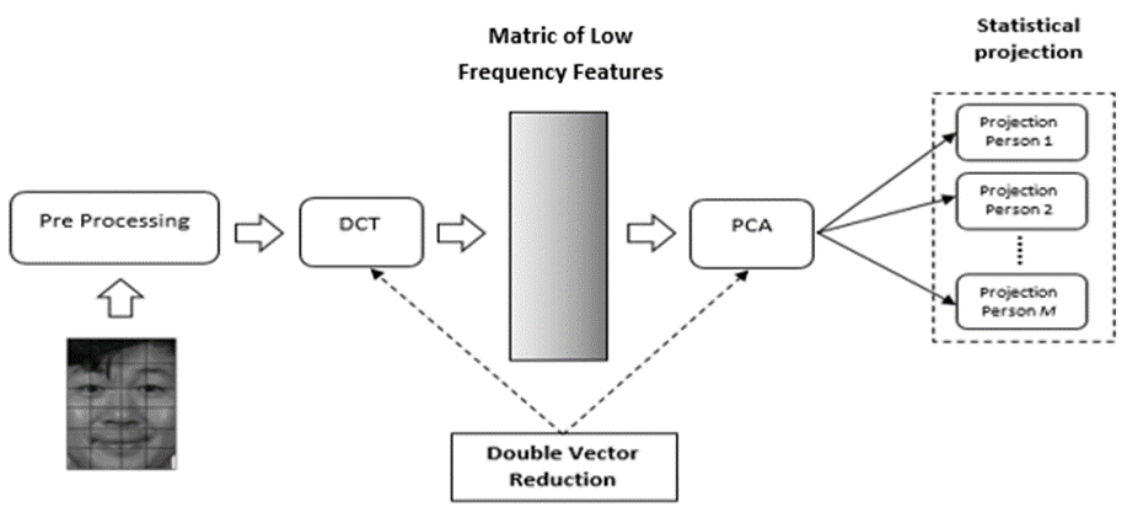

Figure 1. The block diagram of the proposed method implementing new matrix vector of low frequency features and double vector reduction

The advantage of the propose method:

a. Low frequency of DCT coefficients. This approach produces more information which is important for classification and also robust to illumination and pose variations. The extracted features from all block windows are rearrange in a new matrix with respect to the low frequency. 
b. The multiple stage of reduction reduces the size of feature vector. The substantial reduction amount of features will increase the performance over the conventional method.

c. Local features extraction based on DCT are computed in several image regions. This method separates the image into several regions that has different discrimination power. The extracted information in small window will increase computational speed.

The block diagram in Figure 1 shows all the important stage from input image until the classification process. At the pre-processing stage, all images are subdivided into a block window and each window is transformed to the DCT domain. Low frequency features are preserved by selecting several DCT coefficients using zig-zag scanning approach. PCA is then applied in the next stage to optimize the reduction of feature dimension by selecting the most eigenvalues that represent the image. The two stage vector reduction mention above occurred in DCT domain and linier projection method.

\subsection{Extraction of local features}

The feature extraction is important in face recognition as it is used to capture the informative component exist in face image. The extracted features must be valuable to the next process, such as image projection and subject classification with allowable error rate. Furthermore, the feature extraction should be effective with regards to computational resource such as memory and processing time.

Extraction of features can be done in several ways; 1) Holistic. 2) Local feature. Holistic feature generally tends to produce high dimensional feature, which makes it powerless to directly classify in the feature space. Therefore, method such as DCT and PCA are employed in holistic-base method as dimensional reduction techniques [2-4].

Another approach to extract holistic information is feature-based approach such as Wavelet transform [5], Discrete cosine transform [6], and Fourier transform [7]. In this approach the features are extracted from spatial frequency technique. The entire image is converted into the frequency domain then only some coefficients are preserved.

Nowadays several researchers have started to implement local features for biometric recognition. This approach uses several local observations obtained from an image to represent the image features [8, 9]. The advantages to the holistic features it has lower dimensional features, and the local features are more robust to the illumination, variation, and occlusion as spatially in face recognition. Local Binary Pattern (LBP) is one of feature base method which used to examine the pattern of a biometric face representation into histogram.

Another method is component based approach where the images are divided into several blocks. The image from each block are extracted independently and then used for classification process. Several approach using this method are Gaborfeatures was develop by [10], component based LDA [11] and modular PCA [12]. Local features based on frequency coefficient can be extracted using Discrete Cosine Transform. In this method a face image is divided into sub block then another process is performed to extract features from low frequency band. A successful method based on frequency coefficient was developed base on statistical model such as a Principle Component Analysis (PCA) [13] and Linear Discriminant Analysis (LDA) [14].

In this paper, we utilize the local feature based on frequency coefficient extracted using DCT method. Sub block based feature are robust to the illumination and pose variation compared to global features. Furthermore, to archive less computational cost our proposed method only used 3 coefficients from a 28x46 window frame with no overlapping block. The first coefficient is preserve inside the matrix shown in Figure 2. This coefficient is known as DC component which representing the average of an image, while the rest are high frequency components of image. Method in [15] proved that the high frequency information by itself is insufficient for good face recognition performance. The rejection of high frequency component also causes the image to be robust to scale variations which is required in face recognition system. The practically observation and experiment conducted by [16] show the DC coefficient holding $95 \%$ of the energy and proofed the amplitudes are directly related to the energies which carry the information in an image. The general equation for DCT is shown in (1). If an image $f(x, y)$, with dimension $\mathrm{MxN}$ will produce a 2D-DCT $\mathrm{F}(\mathrm{u}, \mathrm{v})$ that has the dimension $\mathrm{MxN}$ and is computed as:

$$
C(u, v)=a_{u} a_{v} \sum_{x=0}^{M-1} \sum_{y=0}^{N-1} f(x, y) \cos \frac{(2 x+1) u \pi}{2 M} \cos \frac{(2 x+1) v \pi}{2 N}
$$

$$
\text { where: } \begin{aligned}
a_{u} & = \begin{cases}\sqrt{1 / M}, & u=0 \\
\sqrt{2 / M}, & 1 \leq u \leq M-1\end{cases} \\
a_{v} & = \begin{cases}\sqrt{1 / N}, & v=0 \\
\sqrt{2 / N}, & 1 \leq v \leq N-1\end{cases}
\end{aligned}
$$




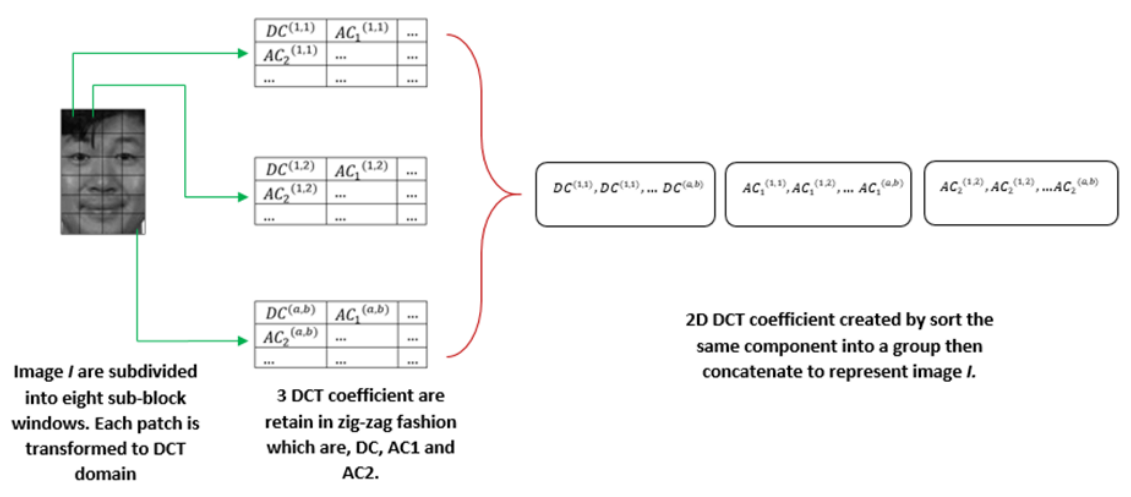

Figure 2. The structure of new matrix of low frequency feature. The components $\left(D C, A C_{1}, A C_{2}\right)$ are group together after extract from all sub-windows, then concatenated to represent face image

\subsection{Double vector reduction}

ORL face database contains a set of face images taken between April 1992 and April 1994 at the lab The images are organized into 40 directories (one for each subject), and Each directory contains 10 different pose of a person. The size of each image is $92 \times 112$ pixels, for a total is 10,304 pixels, with 256 grey levels per pixel.

High dimensional vector caused recognition proses slow and not applicable for low computational resource such DSP board. To avoid the situation occurred we proposed double reduction to reduce; 1) feature vector and 2) feature space.

In DCT stages only three coefficients are selected from each sub-block window and total coefficients used to represent a single face image are 24 which is $0.233 \%$ from the total coefficient size $(10,304)$. This can save a lot of computational resources such as RAM, ROM and processing time. The next features reduction process on PCA stage. Using PCA, the 24 coefficients are reducing by projecting a new dimension space is 10 .

The detail step of PCA are show in (2) until (6). Consider a matrix $N x N$ face image $\Gamma(x, y)$ as a vector of dimension $N^{2}$, so the image can be thought as a point in $N^{2}$ dimensional space. A database of M images can therefore be mapped to a collection of points in this high-dimensional "face space" as $\Gamma_{1}, \Gamma_{2}, \Gamma_{3}, \ldots \Gamma_{M}$

$$
\Psi=\frac{1}{M} \sum_{n=1}^{M} \Gamma_{n}
$$

where: $\mathrm{M}$ is the number of images in the training phase

Compute zero mean image for each train and test images as shown in (3):

$$
\Phi_{i}=\Gamma_{i}-\Psi
$$

where: $i=1,2,3, \ldots, M$

The covariance matrix $\mathrm{C}$ of the data set is defined by (4):

$$
C=\frac{1}{M} \sum_{n=1}^{M} \Phi_{n} \Phi_{n}^{T}=A A^{T}
$$

where: the matrix $A=\left[\Phi_{1}, \Phi_{2}, \ldots \Phi_{M}\right]$.

The next process is to find the $M$ eigenvectors $v_{i}$ of $C$. These vectors determine the linear combination of the $M$ training set face images to form Eigenfaces $u_{i}$. A new face image $(\Gamma)$ is transformed to its eigenface component (i.e., projected into "face space") using the following (6):

$$
\omega_{k}=u_{k}^{T}(\Gamma-\Psi) \mathrm{k}=1,2, \ldots M^{I}
$$

where: $\omega_{k}$ is the $k$-th coordinate of the $\Phi$ 


\subsection{Classification process}

Classification is a process to identify the unknown face which is correlated to the subject face. This process uses both projection test and projection train that are produced by Principle Component Analysis (PCA).

Euclidean distance is one of the simplest and faster classifier as compared to other classifiers. To recognize unknown faces, the Euclidian distance method is used to find the minimum distance between data projection; face tests and the face train. Euclidean distance is defined as the straight-line distance between two points as given in (7). The smallest value of $\epsilon_{-} \mathrm{k}$ distance will make the assumption that they are from the same class.

$$
\epsilon_{k}=\left\|\Omega-\Omega_{k}\right\|^{2}
$$

where: $\Omega^{T}$ describe the $k$-th face class

\section{RESULTS AND ANALYSIS}

The ORL data set consist of 40 persons and contain 10 set of images for each person. The subjects comprise of 4 females and 36 males. For some subject the images were taken with varying lighting, at different times, different poses, facial expressions such as; open/closed eyes, smiling/not smiling, and facial details; with glasses/no glasses. These images include up to $20^{\circ}$ of facial titling and rotation. Some differences in scale and changes in brightness also occur. For the background, all images were taken against a dark homogeneous with the subjects in an upright, frontal position (with tolerance for some side movement). The experiment was split into two stage which is personal computer (PC) and single board computer (SBC) TMS320C6713.

\subsection{PC based experiment}

In first experiment the set is manually select to find which set has the highest and the lowest recognition rate where one image used for test and others images used for train, the DCT coefficient and PCA dimension is fixed to 20. Table 1 shown the comparison Accuracy (\%) and Times (s) for Each Selected Set of Train (Tr) and Test (Ts) Image.

The observation from Table 1 can be conclude that the best accuracy (\%) is $100 \%$ with a lowest time consumption 0.72 seconds (s) is Set Image 7 and the worst accuracy is 95\% with the highest time consumption is $0.75 \mathrm{~s}$ of Set Image 1. The experiment continues with those two selected image (Set Image 7 and Set Image 1) shown in Table 1, where their accuracy is compared based on the different number of DCT coefficients, PCA coefficients and number of training images. this section, it is explained the results of research and at the same time is given the comprehensive discussion.

Table 1. Comparison accuracy and times for each selected set of train and test image

\begin{tabular}{cccccc}
\hline DCT & PCA & $(\%)$ & $(\mathrm{s})$ & $(\mathrm{Ts})$ & $(\mathrm{Tr})$ \\
\hline 20 & 20 & 95 & 0.7508 & 1 & $2,3,4 \ldots 10$ \\
20 & 20 & 95 & 0.7086 & 2 & Except 2 \\
20 & 20 & 100 & 0.7729 & 3 & Except 3 \\
20 & 20 & 97.5 & 0.7099 & 4 & Except 4 \\
20 & 20 & 100 & 0.724 & 5 & Except 5 \\
20 & 20 & 97.5 & 0.7201 & 6 & Except 6 \\
20 & 20 & 100 & 0.7233 & 7 & Except 7 \\
20 & 20 & 97.5 & 0.7148 & 8 & Except 8 \\
20 & 20 & 97.5 & 0.6947 & 9 & Except 8 \\
20 & 20 & 100 & 0.7666 & 10 & Except 10 \\
\hline
\end{tabular}

The objective of second experiment is to find the relation between accuracy, execution time, and the number of training image as shown in Figure 3. From the observation execution time increase when number of training increase, then this will affect the system performance. The highest recognition rate achieved when using nine of training image, which is $100 \%$ (set 7) and $95 \%$ (set1), then decaying when using less of training image. However, the best accuracy rate is important to the system. The nine train images are selected as it is applied in the proposed system. 


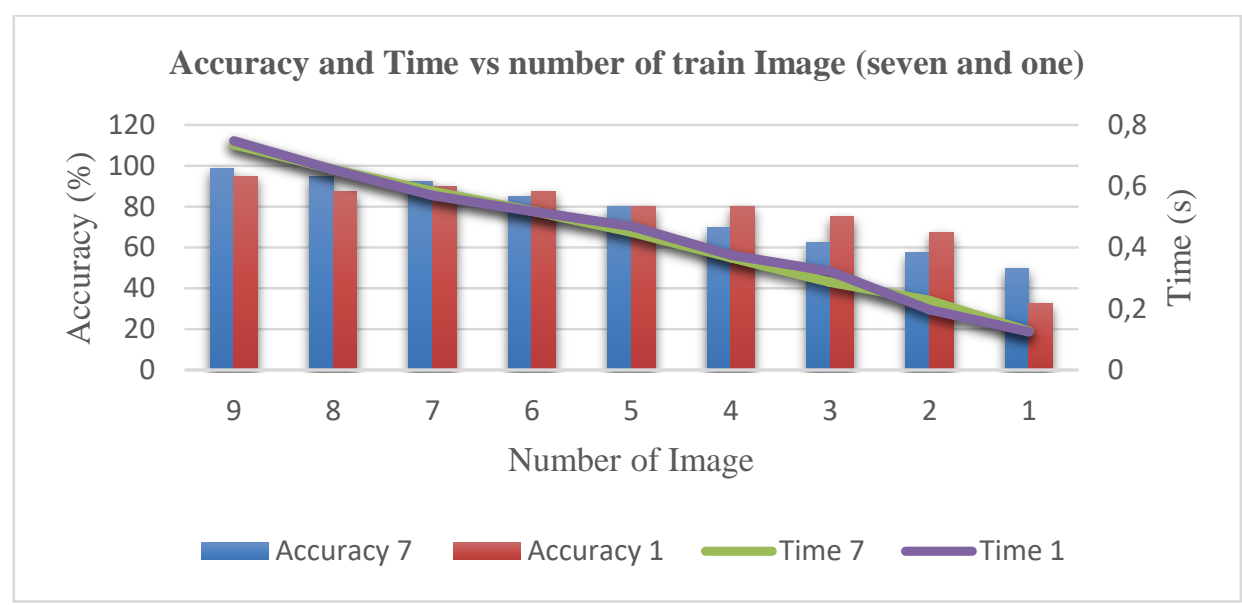

Figure 3. The relationship between accuracy, execution time and number of training image

In the third experiment PCA dimension fixed to 20 and DCT coefficient is varied from 3 to 100 . The result shown in Figure 4. From observation most result remains the same $100 \%$ and $95 \%$ respectively for set 7 and set 5 even if the DCT coefficient is varied from 3 to 100, but the time required to execute the task is increased when more coefficients are used in the process. For best performance and less complex system, the proposed method only 3 DCT coefficients from each sub-block were used.

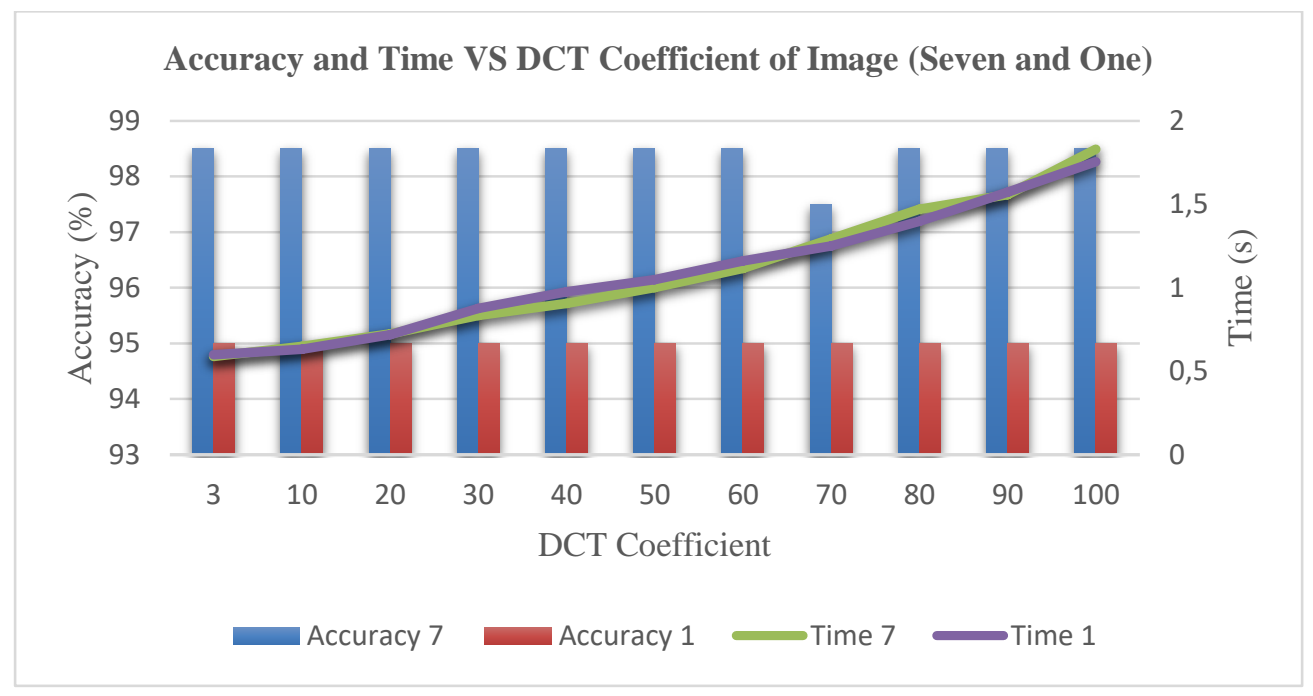

Figure 4. Relationship between accuracy, execution time and DCT coefficient

Figure 5 shows the result of fourth experiment the DCT coefficient is fixed to 20 and then the PCA dimension varies from 1 to 100 . The start of optimum accuracy, which is $100 \%$ for Set Image 7 and $95 \%$ for Set Image 1, is when the PCA dimension equals to 10 and the accuracy maintains until the dimension is 100 . The execution time is proportional to the PCA dimension. The increasing of PCA dimension give more affect system execution time compared to the increasing of DCT coefficient. The execution stime for 100 DCT coefficient versus 100 PCA dimension is 1.8 second and 3.1 second respectively. Based on the analysis result, only 10 of the PCA dimensions are used in the proposed method. 


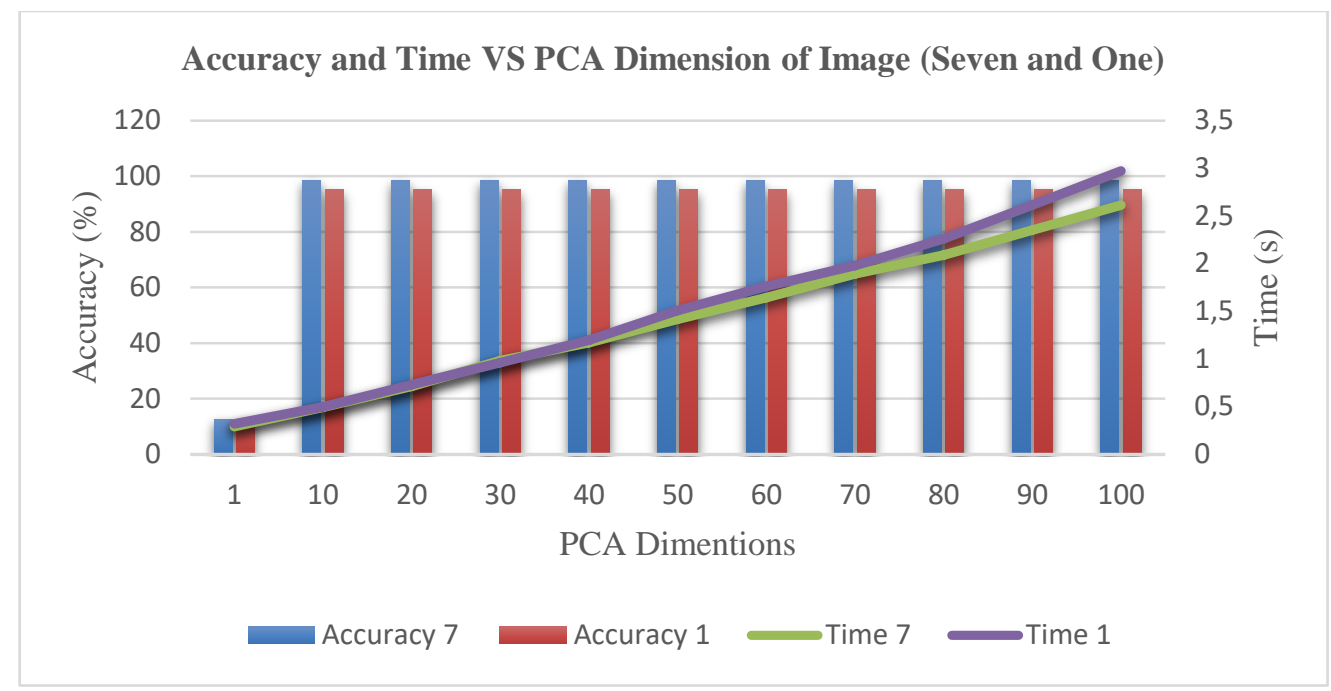

Figure 5. Relationship between accuracy and execution time and PCA dimension

The fifth experiment explains the benefit of local features extraction using DCT compared to holistic extraction using DCT in terms of processing time. From the review of related papers, most of them concluded that local features returns better results than holistic with regards to pose and illumination problems [17, 18]. The result in Figure 6 shown the difference in time always increases proportional to the number of extraction images. In this experiment we try up to five samples, the holistic extraction required 29.24 s longer then local feature.

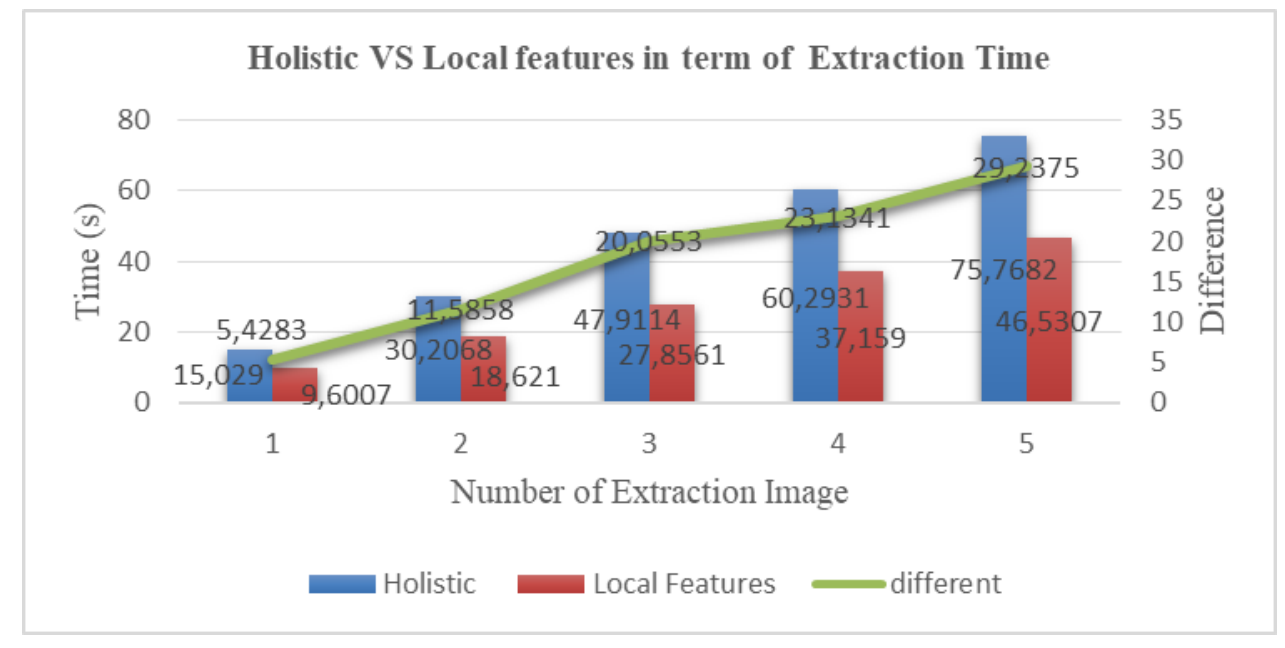

Figure 6. Holistic versus Local features in term of extraction time

The performance of the recognition system that using 3 DCT coefficients from each sub-block image and 10 PCA dimension is tested and detail shown in Table 2. When Set Image 7 is tested, the face recognition performed is $100 \%$ and, when the Set Image 1 is tested, the face recognition performance is $95 \%$ and the recognition process only required around 0.33 s to recognize 40 people.

Table 2. Analysis performance and execution time using 3 coefficients and 10 pca dimension

\begin{tabular}{cccccc}
\hline DCT & PCA & $(\%)$ & $(\mathrm{s})$ & Ts & $\operatorname{Tr}$ \\
\hline 3 & 10 & 100 & 0.33 & 7 & $1,2,3,4,5,6,8,9,10$ \\
3 & 10 & 95 & 0.32 & 1 & $2,3,4,5,6,7,8,9,10$ \\
\hline
\end{tabular}




\subsection{SBC TMS320C6713 based experiment}

In this section, the analysis focused on the TMS320C6713 DSK board to measures the execution time and accuracy. In this experiment only used 30 face images, 3 images used for test and other 27 images used for train.

When both DCT coefficient and PCA dimension are 30, the execution time for PC is $23.96 \mathrm{~ms}$ and $74.24 \mathrm{~ms}$ for the DSP board, and then after reducing the DCT coefficient and PCA dimensions respectively to 3 and 10 . The time for the PC reduces up to $2.17 \mathrm{~ms}$ and the DSP board is reduced to $4.43 \mathrm{~ms}$. The proposed method can reduce a lot of execution time, this can make system efficient and relevant for real time application. The detail of analysis result shown in Table 3.

Table 3. Compare result between DSP board and offline using image set seven

\begin{tabular}{ccccccc}
\hline DCT & PCA & Ts:Tr & SBC (ms) & PC (ms) & SBC (\%) & PC (\%) \\
\hline 30 & 30 & $3: 27$ & 74.24 & 23.96 & 100 & 100 \\
3 & 10 & $3: 27$ & 4.43 & 2.17 & 100 & 100 \\
\hline
\end{tabular}

We believe if we use a DSP board that has the same speed as PC, the execution time for the DSP board is more than five times faster than PC because the PC's using Core i5 with 8 Gigabyte of RAM and measured speed is $2.5 \mathrm{GHz}$. where, DSP board's measured speed is $225 \mathrm{MHz}$. Table 4 shown the comparison of several existing methods with the proposed method.

Table 4. Comparison result of the existing with the top recognition rate of the proposed method

\begin{tabular}{lcc}
\hline \multicolumn{1}{c}{ Methods } & Modalities & Accuracy (\%) \\
\hline Sun et.al.,[21] & ORL & 96 \\
Bozorgtabar et.al.,[20] & ORL & 96.5 \\
Bag et.al.,[19] & ORL & 96.7 \\
Wu FengXiang,[22] & ORL & 98.7 \\
Jawal Nagil et.al.,[23] & ORL & 98.9 \\
Hafiz Imtiaz et.al.,[24] & ORL & 99.75 \\
Proposed method & ORL & 100 \\
\hline
\end{tabular}

\section{CONCLUSION}

Developing a face recognition system is a challenge because the faces always changes due to expression, direction, light, and scale. This paper focuses on the local features extraction approach, which divides the facial image based on the informative zones. The analysis using ORL shows that the result is outperform, which is the highest recognition rate is $100 \%$ for the best selected test image and $95 \%$ for the worst selected test image. Besides that, execution time is to recognize 40 people only requires 0.3313 second. The Image Set 7 and set 1 are used in this experiment to find the best and worth recognition rate, then assume recognition rate of the others set of image are in between this two set. The 95\% mean 38/40 person are correctly recognized. In this experiment, the key of success is training image. Compare to others paper [18-20] they used less of training image instead of nine of training image for each person. However, feature space is still small because the proposed method only extract three of DCT coefficients from each patch then $3 \times 8=24$ coefficients per face image. After PCA reduction only 10 features per a face image used for classification. In other word the system was able to extract and select the valuable features from face image to get high recognition rate, at the same time reduce features space size and less execution time, thus proving this system is simpler, faster, and have a high recognition rate.

\section{ACKNOWLEDGEMENTS}

This research is supported by Ministry of Education Malaysia financial under the Fundamental Research Grant Scheme (FRGS) Grant No: 9003-00583.

\section{REFERENCES}

[1] M. R. Mohd. Zainal, H. Husain, S. A. Samad, and A. Hussain, "Face verification using multiple localized face features," Lect. Notes Comput. Sci. (including Subser. Lect. Notes Artif. Intell. Lect. Notes Bioinformatics), vol. 8237 LNCS, pp. 97-103, 2013.

[2] W. Chen, M. J. Er, and S. Wu, "PCA and LDA in DCT domain," Pattern Recognit. Lett., vol. 26, no. 15, pp. 2474-2482, 2005. 
[3] Z. Fan et al., "Modified Principal Component Analysis: An Integration of Multiple Similarity Subspace Models," in IEEE Transactions on Neural Networks and Learning Systems, vol. 25, no. 8, pp. 1538-1552, Aug. 2014.

[4] N. Kwak, "Principal Component Analysis Based on L1-Norm Maximization," in IEEE Transactions on Pattern Analysis and Machine Intelligence, vol. 30, no. 9, pp. 1672-1680, Sept. 2008.

[5] M. M. Mukhedkar, "Fast Face Recognition Based on Wavelet Transform on PCA," Int. Conf. Energy Syst. Appl. (ICESA 2015), no. Icesa, pp. 761-764, 2015.

[6] S. Dabbaghchian, M. P. Ghaemmaghami, and A. Aghagolzadeh, "Feature extraction using discrete cosine transform and discrimination power analysis with a face recognition technology," Pattern Recognit., vol. 43, no. 4, pp. 1431-1440, 2010.

[7] A. K. Jha, R. Gupta and D. Saini, "Face Recognition: A Fourier Transform and SVD Based Approach," 2013 5th International Conference and Computational Intelligence and Communication Networks, Mathura, 2013, pp. 205-209.

[8] N. Wang, J. Li, D. Tao, X. Li, and X. Gao, "Heterogeneous image transformation," Pattern Recognit. Lett., vol. 34, no. 1 , pp. 77-84, 2013.

[9] N. Wang, D. Tao, X. Gao, X. Li, and J. Li, “A comprehensive survey to face hallucination,” Int. J. Comput. Vis., vol. 106, no. 1, pp. 9-30, 2014.

[10] L. A. Cament, F. J. Galdames, K. W. Bowyer, and C. A. Perez, "Face recognition under pose variation with local Gabor features enhanced by Active Shape and Statistical Models," Pattern Recognit., vol. 48, no. 11, pp. 3371-3384, 2015.

[11] J. A. Dargham, A. Chekima, and M. Hamdan, "Hybrid Component-Based Face Recognition System," Distrib. Comput. Artif. Intell. AISC, no. 151, pp. 573-574, 2012.

[12] Q. Y. Li and H. Y. Quan, "The Dimension Reduction Method of Face Feature Parameters Based on Modular 2DPCA and PCA", Applied Mechanics and Materials, Vols. 687-691, pp. 4037-4041, 2014

[13] K. D. Kadam, "Face Recognition using Principal Component Analysis with DCT," Int. J. Eng. Res. Gen. Sci., vol. 2, no. 4, pp. 276-280, 2014.

[14] S. Fernandes and J. Bala, "Performance Analysis of PCA-based and LDA-based Algorithms for Face Recognition," Int. J. Signal Process. Syst., vol. 1, no. 1, pp. 1-6, 2013.

[15] P. Sinha, B. Balas, Y. Ostrovsky and R. Russell, "Face Recognition by Humans: Nineteen Results All Computer Vision Researchers Should Know About," in Proceedings of the IEEE, vol. 94, no. 11, pp. 1948-1962, Nov. 2006.

[16] M. K, V. Govindarajan, S. K. V V S, and R. S, "Face Recognition using Block-Based DCT Feature Extraction," J. Adv. Comput. Sci. Technol., vol. 1, no. 4, pp. 266-283, 2012.

[17] W. Chen, Meng Joo Er and Shiqian Wu, "Illumination compensation and normalization for robust face recognition using discrete cosine transform in logarithm domain," in IEEE Transactions on Systems, Man, and Cybernetics, Part B (Cybernetics), vol. 36, no. 2, pp. 458-466, April 2006.

[18] X. Zou, J. Kittler and K. Messer, "Illumination Invariant Face Recognition: A Survey," 2007 First IEEE International Conference on Biometrics: Theory, Applications, and Systems, Crystal City, VA, 2007, pp. 1-8.

[19] S. Bag and G. Sanyal, "An efficient face recognition approach using PCA and minimum distance classifier," 2011 International Conference on Image Information Processing, Shimla, 2011, pp. 1-6.

[20] B. Bozorgtabar, H. Azami, and F. Noorian, "Illumination invariant face recognition using fuzzy LDA and FFNN," J. Signal Inf. Process., vol. 2012, no. February, pp. 45-50, 2012.

[21] Guoxia Sun, Liangliang Zhang and Huiqiang Sun, "Face recognition based on symmetrical weighted PCA," 2011 International Conference on Computer Science and Service System (CSSS), Nanjing, 2011, pp. 2249-2252.

[22] F. Wu, "Face Recognition Based on Wavelet Transform and Regional Directional Weighted Local Binary Pattern," J. Multimed., vol. 9, no. 8, pp. 1017-1023, 2014.

[23] J. Nagi, S. K. Ahmed, and F. Nagi, "Pose Invariant Face Recognition using Hybrid DWT-DCT Frequency Features with Support Vector Machines," in Proceedings of the 4th International Conference Information Technology and Multimedia, 2008, no. November, pp. 99-104.

[24] H. Imtiaz and S. A. Fattah, "a face recognition scheme using wavelet based dominant features," Signal Image Prosessing Int. J., vol. 2, no. 3, pp. 69-80, 2011.

\section{BIOGRAPHIES OF AUTHORS}

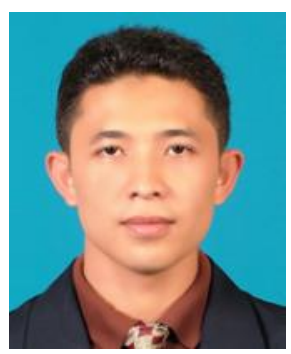

Raja Abdullah Raja Ahmad received the B.Eng. degree in Computer and Communication Engineering from Universiti Malaysia Perlis, M.Sc. degree in Embedded System Design Engineering from Universiti Malaysia Perlis. 


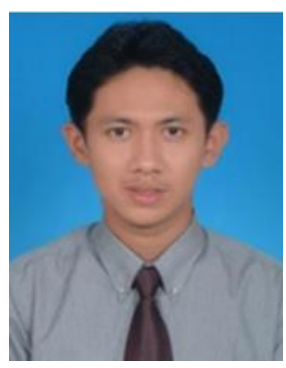

M.I Ahmad received the B.Eng. degree in Electrical and Electronic Engineering from Universiti Teknologi Malaysia, M.Sc. degree in Electronic System Design Engineering from Universiti Sains Malaysia and Ph.D. degree from Newcastle University, Newcastle upon Tyne, UK. He is currently a senior lecturer with the School of Computer and Communication Engineering, Universiti Malaysia Perlis, Malaysia. His current research interests include biometric, information fusion, image processing and pattern recognition.

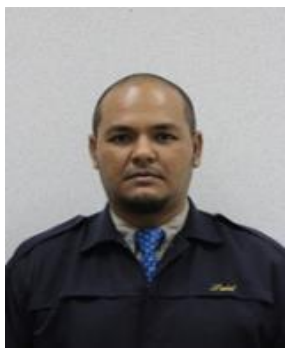

Said Amirul Anwar Ab. Hamid received his $\mathrm{PhD}$ in Computer Engineering from Universiti Sains Malaysia in 2014. Currently he is a senior lecturer at School of Computer and Communication Engineering, Universiti Malaysia Perlis. His research interests include image processing and pattern recognition. Malaysia Perlis, Malaysia. His current research interests include biometric, information fusion, image processing and pattern recognition. 